\title{
Extra Edge Damping as a Way to Improve Sound Insulation of Window Structures
}

\author{
Aleksey Nikolaevich Puzankov, Dmitry Lvovich Shchegolev, Vladimir Aleksandrovich \\ Tishkov and Vladimir Nikolaevich Bobylev
}

Nizhny Novgorod State University of Architecture and Civil Engineering, Nizhny Novgorod, Russia.

\section{(Received 26 September 2016; accepted 9 June 2017)}

The article presents the experimental data of research of sound insulation of window structures using supplementary damping along the perimeter of the translucent portion of the protection with a strip of non-transparent vibration-damping material (edge damping). The authors considered a method for calculating double translucent protective structures with edge damping. They gave versions of possible design solutions for these protective structures.

\section{NOMENCLATURE}

IGU — insulating glass unit

ISO - International Organization for Standardization NNGASU — Nizhegorodsky Gosudarstvenny Arkhitekturnostroitelny Universitet (Nizhny Novgorod State University of Architecture and Civil Engineering)

\section{INTRODUCTION}

Nowadays the increasing sound pollution of urban areas makes it critical to improve sound insulation of outer protective structures of residential and public buildings, particularly those of windows. There are several ways to increase the sound insulation of windows without significantly increasing the material consumption and complexity of the design. Let us consider one of them.

\section{WAYS TO INCREASE SOUND INSULATION USING THE ADDITIONAL DAMPING}

Extra damping of glasses of protection is widely used in modern window structures, and specifically to improve their sound insulation. There are several methods to use extra damping to improve the sound insulation properties of translucent protection:

- method No.1 - damping with a transparent film on the outer surface of the pane;

- method No.2 - damping with a transparent material connecting two layers of the pane (triplex);

- method No.3 - use of non-transparent damping material in certain areas of glazing (e.g., along the perimeter of the structure).

The impact of damping methods No.1 and No.2 has been discussed by many researchers, in particular, by D.V. Murygin, ${ }^{1}$ A.A. Kochkin, ${ }^{2}$ J.G. Lilly, ${ }^{3}$ N. Garg, ${ }^{4}$ U. Keller, ${ }^{5}$ J. Schimmelpenningh ${ }^{6}$ and others. In these and other researches, the results proving the efficiency of these ways of damping for improving sound insulation of various types of translucent enclosing structures are presented.

The third method is now scarcely studied, despite the fact that its application may allow to significantly improve the sound insulation due to the slight reduction of translucent area of the structure. In his paper I.I. Bogolepov ${ }^{7}$ discussed the double polymethylacrylic structure in which a special elastic material applied on the perimeter of the glazing, served for the acoustic separation of protection plates and prevention of occurrence of sound bridges; however, the effect of this material as a damping one has not been discussed. ${ }^{7}$

Currently, there are effective self-adhesive damping materials based on different types of polymer mastics having a loss factor $\eta=0.3-0.4$. It is proposed to use such materials for pasting the perimeter of the transparent portion of the protection. These materials are relatively inexpensive and their costs are low, since the glass area to be covered with these materials is small. Such materials include, in particular, "BiMast Bomb" $(\eta=0.4)$, which is proposed to be used in subsequent experimental studies of the method.

\section{THEORETICAL STUDIES OF POSSIBLE INCREASE OF SOUND INSULATION USING ADDITIONAL DAMPING}

Consider the theoretical possibility of pasting the strip of opaque vibration cushioning material around the perimeter of translucent walling (edge damping) to improve the design of sound insulation.

Since the single-layer translucent structures are practically not used in modern construction practice, the studies on the effect of regional damping on sound insulation of translucent walling should be carried out for the double structure with an air gap.

Theoretical studies of sound insulation of walling of buildings and structures in the NNGASU Laboratory of acoustics are carried out on the basis of the wave fields self-coupling theory (hereinafter - the WFSC theory) developed by a scientific school of Professor M.S. Sedov. ${ }^{8}$ Since there aren't many materials on this subject in English sources, the following ground expressions describing the sound insulation of double walling with an air gap are demonstrated here.

According to the WFSC theory, the walling structure makes a wave motion under the influence of incident sound waves, which involves its own (free) and forced (inertial) waves.

Resonant passage of sound is determined by the degree of the wave fields self-coupling in front of and behind the walling and by the wave field made by own oscillations of the plate. The bigger the self-coupling of sound fields is, the more intense the sound will penetrate through the barrier. Inertial pas- 
sage of sound depends on the surface density of the fence and its geometrical dimensions (length and width).

Given the independence of inertial and free waves in accordance with the principle of superposition of sound transmission, the coefficient for double walling with an air gap can be written as:

$$
\tau=\tau_{s . i .}+\tau_{s . r .}+\tau_{1 i} \tau_{2 i}+\tau_{1 r} \tau_{2 r}
$$

here $\tau_{\text {s.i. }}$ is the coefficient of inertial transmission of sound through the structure as a system of plates with elastic connection between them;

$\tau_{\text {s.r. }}$ is the coefficient of resonant transmission of sound through the structure as a system of plates with elastic connection between them;

$\tau_{1 i}$ is the coefficient of inertial transmission of sound through the first plate;

$\tau_{2 i}$ is the coefficient of inertial transmission of sound through the second plate;

$\tau_{1 r}$ is the coefficient of resonant transmission of sound through the first plate;

$\tau_{2 r}$ is the coefficient of resonant transmission of sound through the second plate.

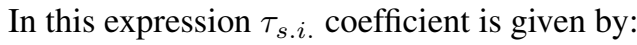

$$
\tau_{\text {s.i. }}=\frac{1}{\frac{\pi^{2}}{\rho_{0}^{2} c_{0}^{2}} \frac{m^{\prime 2} f^{2}}{F_{1 i . a v}^{2}}\left(\frac{f^{2}}{f_{0}^{2}}-1\right)+1} ;
$$

where $m^{\prime}$ is the area density of the walling:

$$
m^{\prime}=m_{1}^{\prime}+m_{2}^{\prime}
$$

wherein $m_{1}^{\prime}$ is the area density of the first walling plate, $\mathrm{kg} / \mathrm{m}^{2}$;

$m_{2}^{\prime}$ is the area density of the second walling plate, $\mathrm{kg} / \mathrm{m}^{2}$;

$f$ is the frequency, $\mathrm{Hz}$;

$\rho_{0} c_{0}$ is the characteristic impedance;

$f_{0}$ is the resonant frequency of the walling, as a system of "mass-elasticity-mass", Hz.

$$
f_{0}=60 \sqrt{\frac{m_{1}^{\prime}+m_{2}^{\prime}}{d m_{1} m_{2}}} .
$$

In this expression, $d$ is the distance between the plates of the walling (the width of the air gap), $\mathrm{m}$.

$F_{1 i}$ is the response function of the first plate, on which the sound impinges.

$$
F_{1 i}=\frac{G_{m n}}{Q_{m n}}
$$

Herein:

$$
\begin{gathered}
G_{m n}=\left\{Q_{m}-\frac{\sin m \pi}{\pi \cdot\left(\mathrm{m}^{2}+m_{1}^{2}\right)} \cdot\left[m_{1} \cdot \sin m \pi+\right.\right. \\
\left.\left.+m \cdot e^{-m_{1} \pi}+m \cdot \beta_{a} \cdot\left(1+e^{-2 \cdot m_{1} \pi}\right)\right]\right\} \times \\
\times\left\{Q_{n}-\frac{\sin n \pi}{\pi \cdot\left(n^{2}+n_{1}^{2}\right)} \cdot\left[n_{1} \cdot \sin n \pi+n \cdot e^{-n_{1} \pi}+\right.\right. \\
\left.\left.+n \cdot \beta_{b} \cdot\left(1+e^{-2 \cdot n_{1} \pi}\right)\right]\right\} ; \\
\beta_{a}=\frac{e^{-m_{1} \pi}-\cos m \pi}{1-e^{-2 \cdot m_{1} \pi}} ; \\
\beta_{b}=\frac{e^{-n_{1} \pi}-\cos n \pi}{1-e^{-2 \cdot n_{1} \pi}} ;
\end{gathered}
$$

$$
\begin{gathered}
m=\frac{k_{0} \cdot a}{\pi} \cdot \cos \alpha_{x} ; \\
n=\frac{k_{0} \cdot b}{\pi} \cdot \cos \alpha_{y} ; \\
\cos \alpha_{x}=\frac{b \cdot \sin \theta_{a v}}{\sqrt{a^{2}+b^{2}}} ; \\
\cos \alpha_{y}=\frac{a \cdot \sin \theta_{a v}}{\sqrt{a^{2}+b^{2}}} ; \\
m_{1}=\frac{k_{0} \cdot a}{\pi} \cdot \sqrt{1+\sin ^{2} \alpha_{x}} ; \\
n_{1}=\frac{k_{0} \cdot b}{\pi} \cdot \sqrt{1+\sin ^{2} \alpha_{y}} ; \\
\cos ^{2} \alpha_{x}+\cos { }^{2} \alpha_{y}+\cos { }^{2} \theta=1 ; \\
Q_{m n}=Q_{m} \cdot Q_{n} ; \\
Q_{m}=\frac{1}{2} \cdot\left(1+\frac{\sin 2 m \pi}{2 m \pi}\right) ; \\
Q_{n}=\frac{1}{2} \cdot\left(1+\frac{\sin 2 n \pi}{2 n \pi}\right) .
\end{gathered}
$$

Here:

$a$ and $b$ is the walling dimensions on the plan (length and width), m;

$\alpha_{x}$ and $\alpha_{y}-$ slip angles of the sound wave along the sides of the plate $a$ and $b$;

$k_{0}$ - the wave number of the medium, $m^{-1}$;

$\theta_{a v}$ - the angle of incidence of the sound waves at the first walling plate (in diffused falling $\theta_{a v}=51.7575^{\circ}$ ).

The coefficient $\tau_{\text {s.r. }}$ in the expression (1) is defined by:

$$
\tau_{\text {s.r. }}=\frac{1}{\frac{\pi^{2}}{\rho_{0}^{2} c_{0}^{2}} \frac{m^{\prime 2} f^{2}}{A^{2}}\left(\frac{f^{2}}{f_{0}^{2}-1}\right)+1} ;
$$

where $A$ is the characteristic of self-coupling.

The value of characteristic of self-coupling depends on the field frequency range. Boundary frequencies of the areas of simple spatial resonance (hereinafter - SSR), incomplete spatial resonance (hereinafter - the ISR) and the full spatial resonance (hereinafter - FSR) is calculated by the following expressions.

Boundary frequency of SSR:

$$
f_{r m_{0} n_{0}}=\frac{c_{0}}{4 \cdot a}
$$

where $c_{0}$ is the speed of sound in air, $\mathrm{m} / \mathrm{s}$.

This area is characterized by a mismatch between the speed vectors of sound waves in the plane of the plate and the own elastic waves.

Boundary frequency of ISR:

$$
f_{r m n_{0}}=\frac{c_{0}}{2 \cdot a \cdot \sin \alpha_{m n_{0}}}
$$

where

$$
\sin \alpha_{m n_{0}}=\frac{b}{\sqrt{\frac{a^{2}}{4}+b^{2}}} .
$$

$\alpha_{m n_{0}}$ is the angle between the sound waves impinging on the plate and waves of own oscillations of the plate.

In the ISR area the characteristics of own elastic and acoustic waves are not fully coincide: the speed of the spread of 
tracks of free and sound waves on one side of the plate are equal to each other, and on the other side they are in such a ratio, in which the response of the plate is largest.

Boundary frequency of FSR:

$$
f_{r m n}=\frac{c_{0}^{2}}{2 \pi} \sqrt{\frac{m_{1}^{\prime}}{D_{1}}} .
$$

In this frequency range the conditions of complete selfcoupling of sound fields in front of and behind the walling structure and the wave field of the natural oscillations of the walling are met.

In the SSR range at frequencies below the boundary frequency of the ISR $\left(f<f_{r m n_{0}}\right)$ the expression for the selfcoupling characteristic has the following form:

$$
A_{0 m_{0} n_{0}}^{2}=\Delta N_{P} \frac{m_{c p}^{4}}{\left(m_{a v}^{2}-m_{0 a v}^{2}\right)^{4}} ;
$$

where $\Delta N_{P}$ is the number of resonances in the frequency band $\Delta f$ :

$$
\Delta N_{P}=\frac{\Delta f a b}{2 \sqrt{\frac{D_{1}}{m_{1}^{\prime}}}}
$$

here: $\Delta f=f_{u}-f_{d}, \mathrm{~Hz}$

$f_{d}$ is the lower boundary frequency, $\mathrm{Hz}$;

$f_{u}$ is the upper boundary frequency, $\mathrm{Hz}$.

The values of $f_{d}$ and $f_{u}$ are calculated by the expressions:

$$
f_{d}=\sqrt{f_{n} f_{n-1}} ; f_{u}=\sqrt{f_{n} f_{n+1}} ;
$$

where $f_{n}$ is the center frequency of the current one-third octave band, $\mathrm{Hz} ; f_{n+1}$ is the center frequency of the following one-third octave band, $\mathrm{Hz} ; f_{n-1}$ is the center frequency of the previous one-third octave band, $\mathrm{Hz}$;

$D_{1}$ is bending stiffness of the first walling plate, defined by the relation:

$$
D_{1}=\frac{E h^{3}}{12\left(1-\sigma^{2}\right)}
$$

where $\sigma$ is the Poisson's ratio;

$E$ is the elastic modulus, $\mathrm{Pa}$;

$h$ is the thickness of the walling plates, $\mathrm{m}$.

$$
\begin{aligned}
& m_{a v}^{2}=\frac{2 f b^{2}}{\pi \sqrt{\frac{D_{1}}{m_{1}^{\prime}}}\left(1+\frac{b^{2}}{a^{2}}\right)} \\
& m_{0 a v}^{2}=\frac{1}{\pi^{8}}\left(\frac{f a b}{\sqrt{\frac{D_{1}}{m_{1}^{\prime}}}}\right)^{2} .
\end{aligned}
$$

In the ISR range the frequency range the $\left(f_{r m n_{0}}<f<\right.$ $\frac{f_{r m n}}{2}$ ) expression for the self-coupling characteristic will be:

$$
A_{0}^{2}=m_{0 \max } \frac{n^{2}}{\left(n^{2}-n_{0 a v}^{2}\right)^{2}}+n_{0 \max } \frac{\mathrm{m}^{2}}{\left(\mathrm{~m}^{2}-m_{0 a v}^{2}\right)^{2}} .
$$

In this expression:

$$
m_{0 \max }=a \sqrt{\frac{4 f^{2}}{c_{0}^{2}}-\frac{1}{b^{2}}}
$$

$$
\begin{gathered}
n_{0 \max }=b \sqrt{\frac{4 f^{2}}{c_{0}^{2}}-\frac{1}{a^{2}}} ; \\
n_{0 a v}^{2}=\left(\frac{n_{0 \max }}{2}\right)^{2} ; \\
m_{0 a v}^{2}=\left(\frac{m_{0 \max }}{2}\right)^{2} ; \\
n^{2}=b^{2}\left(\frac{2}{\pi} \sqrt{\frac{m^{\prime}}{D}} f-\frac{m_{0 a v}^{2}}{a^{2}}\right) ; \\
\mathrm{m}^{2}=a^{2}\left(\frac{2}{\pi} \sqrt{\frac{m^{\prime}}{D}} f-\frac{n_{0 a v}^{2}}{b^{2}}\right) .
\end{gathered}
$$

In the frequency range $\left(\frac{f_{r m n}}{2}<f<f_{r m n}\right)$, the selfcoupling characteristic is given by:

$$
A_{00}^{2}=A_{0}^{2}+A_{01}^{2} ;
$$

where the value $A_{0}^{2}$ is defined by the expression (17), and the additional value of $A_{01}^{2}$ is:

$$
\begin{aligned}
A_{01}^{2}=m_{01 \max } \cdot \frac{n_{1}^{2}}{\left(n_{1}^{2}-n_{01 a v}^{2}\right)^{2}}+ & \\
& +n_{01 \max } \cdot \frac{m_{1}^{2}}{\left(m_{1}^{2}-m_{01 a v}^{2}\right)^{2}} .
\end{aligned}
$$

Herein:

$$
\begin{aligned}
& m_{1}^{2}=n_{1}^{2}=\frac{2 f}{\pi}\left(\frac{1}{a^{2}}+\frac{1}{b^{2}}\right) \sqrt{\frac{D}{m^{\prime}}} \\
& m_{01 \text { max }}=a \sqrt{\frac{4 f^{2}}{c_{0}^{2}}-\frac{m_{1}^{2}}{b^{2}}} \\
& n_{01 \max }=b \sqrt{\frac{4 f^{2}}{c_{0}^{2}}-\frac{n_{1}^{2}}{a^{2}}} \\
& n_{01 a v}^{2}=\left(\frac{n_{01 \max }}{2}\right)^{2} \\
& m_{01 a v}^{2}=\left(\frac{m_{01 \max }}{2}\right)^{2}
\end{aligned}
$$

The coefficients $\tau_{1 i}$ and $\tau_{2 i}$ (1) describe the sound wave passing sequentially through the first plate, the air gap and the second plate. They are determined by the expressions:

$$
\begin{aligned}
\tau_{1 i}= & \frac{1}{\frac{\pi^{2}}{\rho_{0}^{2} c_{0}^{2}} \frac{m_{1}^{\prime 2} f^{2} \cos ^{2} \theta_{a v}}{F_{1 i . a v}^{2}}+1} \\
\tau_{2 i} & =\frac{1}{\frac{\pi^{2}}{\rho_{0}^{2} c_{0}^{2}} \frac{m_{1}^{\prime 2} f^{2} \cos ^{2} \theta_{2}}{F_{2 i . a v}^{2}}+1}
\end{aligned}
$$

Here $\theta_{2}$ is the angle of incidence of sound waves constituting the natural vibration shape of the air gap along the plane of the second plate:

$$
\cos \theta_{2}=\frac{d}{\sqrt{\frac{a^{2}+b^{2}}{2}+d^{2}}} .
$$

The nature of the resonance passage through the first and second plates is different for the following frequency areas: 


$$
f<\frac{f_{r m n}}{2} ; \frac{f_{r m n}}{2}<f<f_{r m n} ; f>f_{r m n} .
$$

In the frequency range, the $\left(f<\frac{f_{r m n}}{2}\right)$ coefficients of resonant passage from the expression (1) have the form:

for the first walling plate:

$$
\tau_{1 r}=\frac{1}{\frac{1.15 \pi^{3}}{8 \rho_{0}^{2} c_{0}^{2} A_{01}^{4}} m_{2}^{\prime 2} f^{2} \eta_{1} \cos ^{2} \theta_{a v}+1} ;
$$

for the second walling plate:

$$
\tau_{2 r}=\frac{1}{\frac{1.15 \pi^{3}}{8 \rho_{0}^{2} c_{0}^{4} A_{02}^{4}} m_{2}^{\prime 2} f^{2} \eta_{2} \cos ^{2} \theta_{2}+1} .
$$

Here $\eta_{1}, \eta_{2}$ are the loss factor for the first and second plates, respectively.

For the frequency range $\left(\frac{f_{r m n}}{2}<f<f_{r m n}\right)$ the expressions (22) and (23) take the form:

for the first walling plate:

$$
\tau_{1 r}=\frac{1}{\frac{1.15 \pi^{3}}{8 \rho_{0}^{2} c_{0}^{2} A_{001}^{4}} m_{2}^{\prime 2} f^{2} \eta_{1} \cos ^{2} \theta_{a v}+1} ;
$$

for the second walling plate:

$$
\tau_{2 r}=\frac{1}{\frac{1.15 \pi^{3}}{8 \rho_{0}^{2} c_{0}^{2} A_{001}^{4}} m_{2}^{\prime 2} f^{2} \eta_{2} \cos ^{2} \theta_{2}+1} ;
$$

For the FSR area for the frequencies $\left(f>f_{r m n}\right)$ the coefficients of the resonant sound passage for the first and second plates are, respectively:

$$
\begin{gathered}
\tau_{1 r}=\frac{1}{\frac{8 \pi}{\rho_{0}^{2} c_{0}^{2}} m_{1}^{\prime 2} \frac{f^{3}}{f_{r m n}} \eta_{1} \cos ^{2} \theta_{a v} \sqrt{1-\frac{f_{r m n}}{f}}+1} ; \\
\tau_{2 r}=\frac{1}{\frac{8 \pi}{\rho_{0}^{2} c_{0}^{2}} m_{2}^{\prime 2} \frac{f^{3}}{f_{r m n}} \eta_{2} \cos ^{2} \theta_{2} \sqrt{1-\frac{f_{r m n}}{f}}+1} .
\end{gathered}
$$

In the above expressions the damping of the vibrations in the walling plate is taken into account by the coefficient of the material losses $\eta$. Thus, it can be concluded that the additional damping affects the resonant oscillations of the walling plates to which it is applied.

To assess the impact of the edge damping on sound insulation of the structure it is proposed to introduce in the expressions (22)-(27) the value of the effective loss coefficient of construction $\left(\eta_{e f 1}, \eta_{e f 2}\right)$. The effective loss coefficient is the rate of glass losses considering the damping with fragments of vibration cushioning material. Changing the effective loss coefficient will have an impact on the coefficients of the resonant transmission of sound: $\tau_{1 r}$ and $\tau_{2 r}$.

The values of $\eta_{e f}$ for glass with partial pasting with damping material can be measured experimentally by the Oberst method (standard method to measure the loss factor of the material). ${ }^{9}$ The results of measurements for glass $4 \mathrm{~mm}$ thick with different pasting area of vibration cushioning material "BiMast Bomb" conducted at the Acoustics Laboratory of NNSUACE are presented in Table 1.

As can be seen from the data shown in the table, the effective loss coefficient in case of the edge damping with the
Table 1. The results of measurements of the effective loss factor for a glass $4 \mathrm{~mm}$ thick

\begin{tabular}{|c|c|}
\hline $\begin{array}{c}\text { Sample area } \\
\text { covered with vibration } \\
\text { cushioning material, } \%\end{array}$ & $\begin{array}{c}\text { The effective loss factor } \eta_{\text {ef }} \\
\text { measured according } \\
\text { to the Oberst } \text { method }^{9}\end{array}$ \\
\hline 0 & 0.006 \\
\hline 12.5 & 0.019 \\
\hline 25 & 0.022 \\
\hline 50 & 0.023 \\
\hline 100 & 0.026 \\
\hline
\end{tabular}

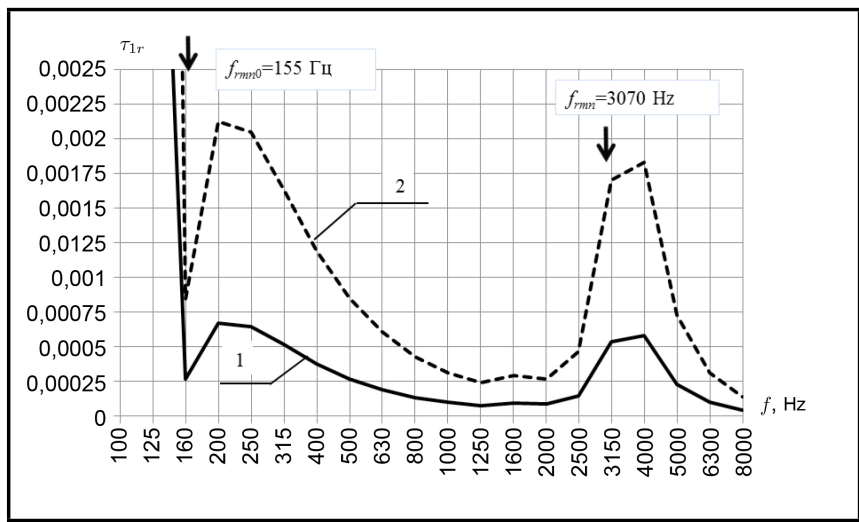

Figure 1. Comparison of values of coefficient $\tau_{1 r}$ when the loss factor: $1-$ $\eta_{\text {ef }}=0.006 ; 2-\eta_{e f}=0.019$.

area $12.5 \%$ of structure area exceeds the loss coefficient of the glass by 3 times. It is proposed to consider the damping area of $12.5 \%$ as the most effective value, since with this relatively small pasting area a significant increase in the effective loss rate is achieved, and with further increase in the area of vibration cushioning material a significant increase in the values of the effective loss coefficient does not occur.

In order to estimate the expected increase in sound insulation due to the use of regional damping by the above procedure, there has been calculated the coefficients $\tau_{1 r}$ and $\tau_{2 r}$ and soundproofing coefficient $\mathrm{R}, \mathrm{dB}$ for the double walling of silica glass of a size $1.3 \times 1.1 \mathrm{~m}$ with a glazing formula $4+24+$ $4 \mathrm{~mm}$ (two sheets of silicate glass $4 \mathrm{~mm}$ thick each, separated by an air gap of $24 \mathrm{~mm}$ width). The calculation was made for two values of the effective loss factor:

1. $\eta_{e f}=0.006$ (glass without additional damping);

2. $\eta_{e f}=0.019$ (glass with an additional damping).

The calculation results are shown in Figures 1,2, 3 .

As seen from frequency characteristics in Fig. 1 and Fig. 2, with an increase in the loss factor from 0.006 to 0.019 the values of coefficients $\tau_{1 r}$ and $\tau_{2 r}$ significantly increase over the entire frequency range.

However, according to the WFSC theory the impact of $\tau_{1 r}$ and $\tau_{2 r}$ on sound insulation is largely dependent on the frequency range. These coefficients have the greatest impact on the structure sound insulation at frequencies $f>\frac{f_{r m n}}{2}$. At frequencies below $\frac{f_{r m n}}{2}$ the effect of $\tau_{1 r}$ and $\tau_{2 r}$ is slightly less (the inertial passage of sound has more influence in this case). At frequencies close to the resonance frequency of the structure as a system of "mass-elasticity-mass" $f_{0}$, the influence of these coefficients is minimal. The sound insulation here is mainly determined by coefficients, that considering the construction as a whole: $\tau_{\text {s.i. }}$ and $\tau_{\text {s.r. }}$.

Thus, for walling with glazing formula $4+24+4 \mathrm{~mm}$, we should expect a substantial increase of sound insulation at frequencies above $1600 \mathrm{~Hz}$, and a slight increase in the range of $315-1600 \mathrm{~Hz}$, which is confirmed by calculating the insulation 


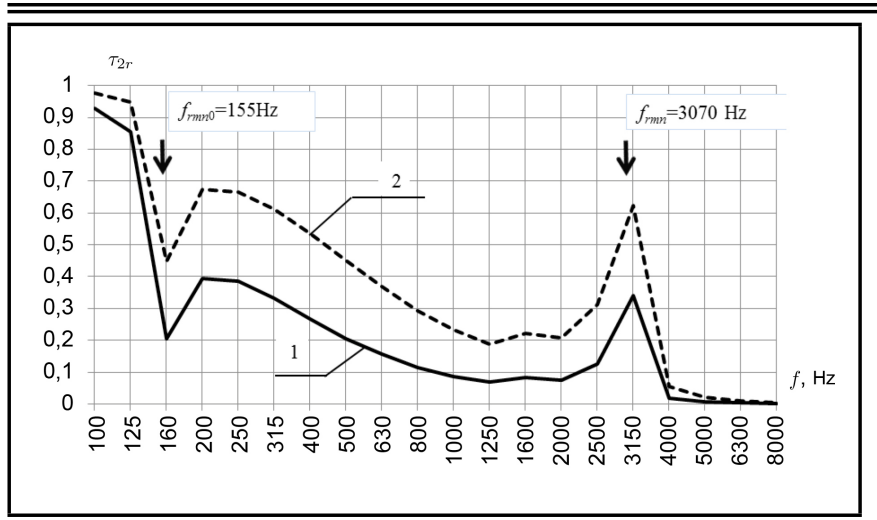

Figure 2. Comparison of values of the coefficient $\tau_{2 r}$ when the loss factor: 1 $-\eta_{e f}=0.006 ; 2-\eta_{e f}=0.019$.

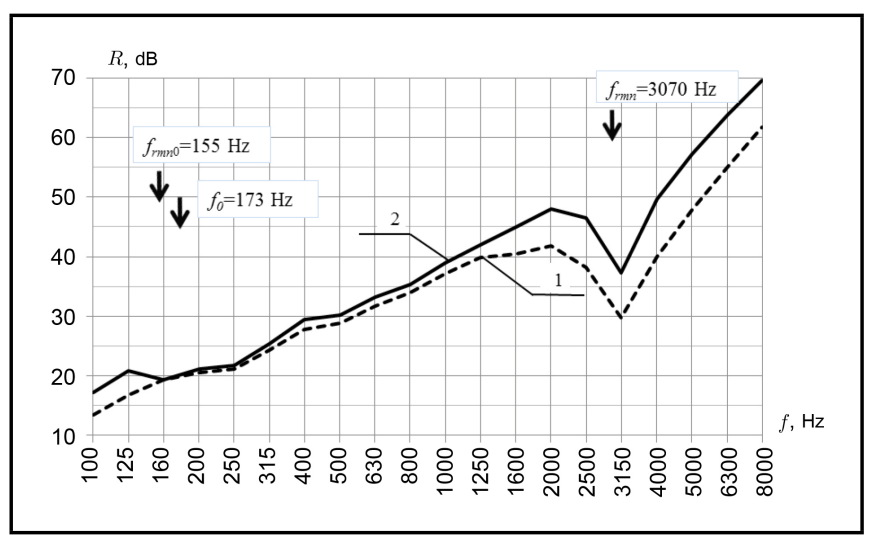

Figure 3. Comparison of the calculated values of the walling insulation with the glazing formula $4+24+4 \mathrm{~mm}$ at: $1-\eta_{e f}=0.006 ; 2-\eta_{e f}=0.019$.

shown in Fig. 3.

To test the assumptions, an experimental study of the effect of additional edge damping on sound insulation in the translucent double walling with an air gap in the NNGASU Laboratory of acoustics was carried out.

\section{DESCRIPTION OF STUDIES' METHODS \\ EXPERIMENTAL}

The sound insulation of studied protection structures has been studied in large reverberant chambers of the NNGASU Laboratory of acoustics according to standard procedure ISO 10140-2 «Acoustics - Laboratory measurement of sound insulation of building elements - Part 2: Measurement of airborne sound insulation». ${ }^{10}$

The measurements were made using a precision acoustic instrumentation "Larson \& Davis" (2900V spectrum analyzer, $1 / 2^{\prime \prime}$ measuring microphones, type 2559). Measuring microphones were sequentially installed in eight points in high- and low-level chambers.

In the high-level chamber of $150 \mathrm{~m}^{3}$ with the help of soundamplifying equipment the sound pressure levels in the range of $100-110 \mathrm{~dB}$ were created. In the low-level chamber of $66 \mathrm{~m}^{3}$ the exceedance of the valid signals over intrinsic noise was more than $15 \mathrm{~dB}$. This condition is satisfied for all frequencies of the test range (100-8000 Hz of the 1/3-octave RMS amplitude spectrum). The lower limit of the frequency range is due to boundary frequency of the diffuseness of reverberation chambers in NNGASU Laboratory of acoustics.

The average sound pressure levels $\left(L_{m}, \mathrm{~dB}\right)$ in the measuring chambers were determined by the formula 10 :

$$
L_{m}=10 \cdot \lg \left(\frac{1}{n} \sum_{i=1}^{n} 10^{0.1 \cdot L_{i}}\right)
$$

where $n$ is the number of measuring points in the measuring chambers (in this case $n=8$ for each chamber);

$L_{i}, \mathrm{~dB}$ is the sound pressure level in the $i$ th point.

Sound insulation of the enclosing structures $(R, \mathrm{~dB})$ when exposed to air noise was calculated by the formula 10 :

$$
R=L_{m 1}-L_{m 2}+10 \cdot \lg \frac{S}{A}
$$

where $L_{m 1} \cdot L_{m 2}, \mathrm{~dB}$ - the average sound pressure levels in a high level chamber and in the low level chamber, respectively;

$S, \mathrm{~m}^{2}$ - the area of the tested enclosure;

$A=\frac{0,16 \cdot V}{T}, \mathrm{~m}^{2}-$ the equivalent acoustic absorption area of low-level camera;

$V, \mathrm{~m}^{3}$ - the volume of low level chamber;

$T, \mathrm{~s}-$ the average time of reverberation in the low level chamber.

For each tested type of the enclosing structures, the measurement of sound insulation was carried out three times. Accuracy of measurements was $\pm 1.0 \mathrm{~dB}$.

\section{THE DESCRIPTION OF STUDIED TRANSLUCENT WALLING}

The translucent structures based on insulated glass units are widely used in construction practice, so to conduct a research on the effect of the edge damping on the sound insulation of double walling the insulated glass unit was manufactured with the glazing formula $4+24+4 \mathrm{~mm}$ with plan dimensions of $1.3 \times 1.1 \mathrm{~m}$.

The specific material "BiMast Bomb" manufactured by "Standartplast" LLC (Russia) was used for additional edge damping. This material has one of the highest damping characteristics $(\eta=0.4)$ among opaque self-adhesive damping materials, that are common in the Russian market.

The sound insulation of double glazed unit with varying degrees of regional damping was measured in the course of these experimental studies.

The scheme of the studied structure is presented in Figure 4.

\section{RESULTS OF THE PERFORMED EXPERIMENTAL STUDIES}

Frequency characteristics obtained during the experiment are presented in Figure 5. The experimental results confirm the data of a theoretical calculation of the frequency range in which can be expected the increased sound insulation of the structure when using edge damping.

The main gain in sound insulation when applying edge damping occurs in the frequency range $315-3150 \mathrm{~Hz}$. The most effective way to improve sound insulation proved to be damping both panes of glass with strip material "BiMast Bomb" $40 \mathrm{~mm}$ wide around the perimeter (at that, area occupied by the vibration cushioning material is $13 \%$ of the total area of glazing structure), which roughly corresponds to the "effective" area of damping material $12.5 \%$ obtained by determining the effective loss factor. The main gain in sound insulation is $2-8 \mathrm{~dB}$ when applying edge damping in the frequency range $315-3150 \mathrm{~Hz}$. In the frequency range below $315 \mathrm{~Hz}$ the 


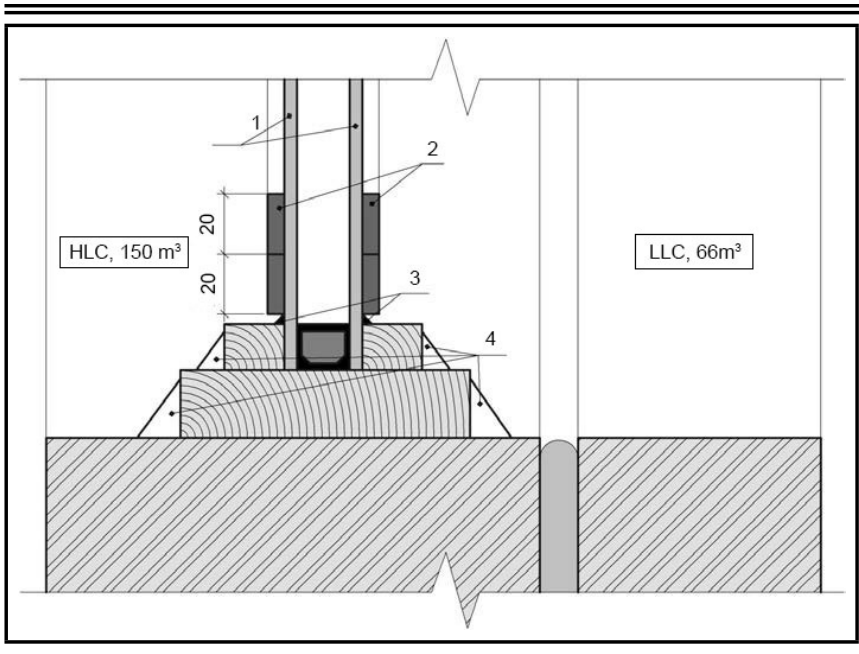

Figure 4. The scheme of the studied structure, as set out in the opening of large reverberant chambers of Acoustics Laboratory of NNGASU: 1 - glass unit with glazing formula $4+24+4 \mathrm{~mm} ; 2$ - vibration cushioning material "BiMast Bomb"; 3 — silicone sealant; 4 — plaster rim.

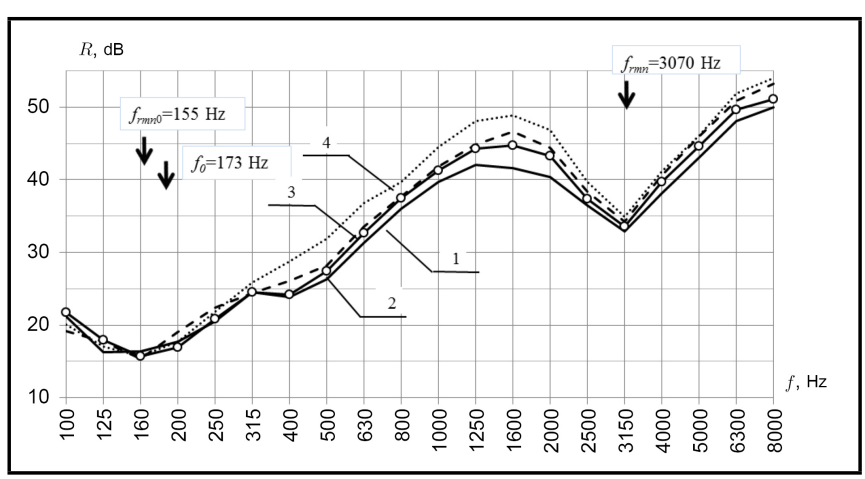

Figure 5. Sound insulation of glass unit $4+24+4 \mathrm{~mm}$ with varying degrees of edge damping material "BiMast Bomb" (experimental data): 1 - without the use of edge damping; $2-20 \mathrm{~mm}$ damping along the perimeter (single glass); 3 - $20 \mathrm{~mm}$ damping along perimeter (both glasses); $4-40 \mathrm{~mm}$ damping along the perimeter (both glasses).

visible gain in sound insulation is not observed. With a smaller area of vibration damping material the gain in sound insulation is much less, but there it is observed in the same frequency range.

The lack of increase in sound insulation at frequencies below $315 \mathrm{~Hz}$ supports the conclusion that the additional damping is inefficient at frequencies close to the resonance frequency of "mass-elasticity-mass".

It is necessary to draw attention to the fact that the application of the regional damping leads to increase in weight of the structure. However, this mass change is very small and does not exert any significant influence on the sound insulation of enclosure. For the investigated structure with damping using $40 \mathrm{~mm}$ wide strip around the perimeter of both glasses, the weight is increased by $12 \%$ of the original weight of the glazing without damping.

It should also be noted that the design of double translucent enclosing structures based on glass units provides the additional damping element by applying a sealing material for insulating glass perimeter. However, as follows from the results of the experiment, the additional edge damping improves the sound insulation even in this case.

Also the additional damping arising during construction of the measuring aperture can influence the sound insulation of the structure. However, during these measurements the method of installing glass in the structure did not change from mea-

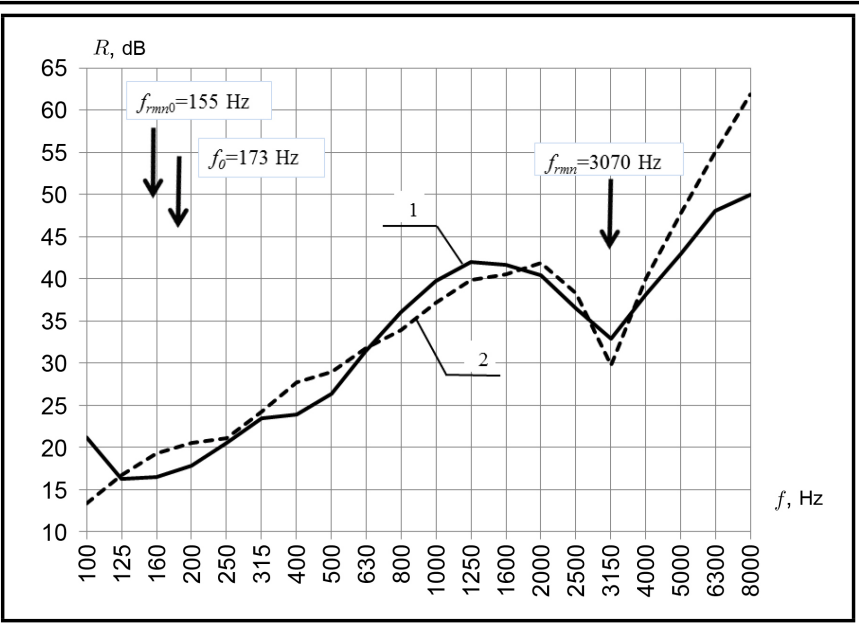

Figure 6. Frequency characteristics of sound insulation of double-glazed unit of $4+24+4 \mathrm{~mm}$ without the edge damping: 1 - experimental data; $2-$ theoretical calculation.

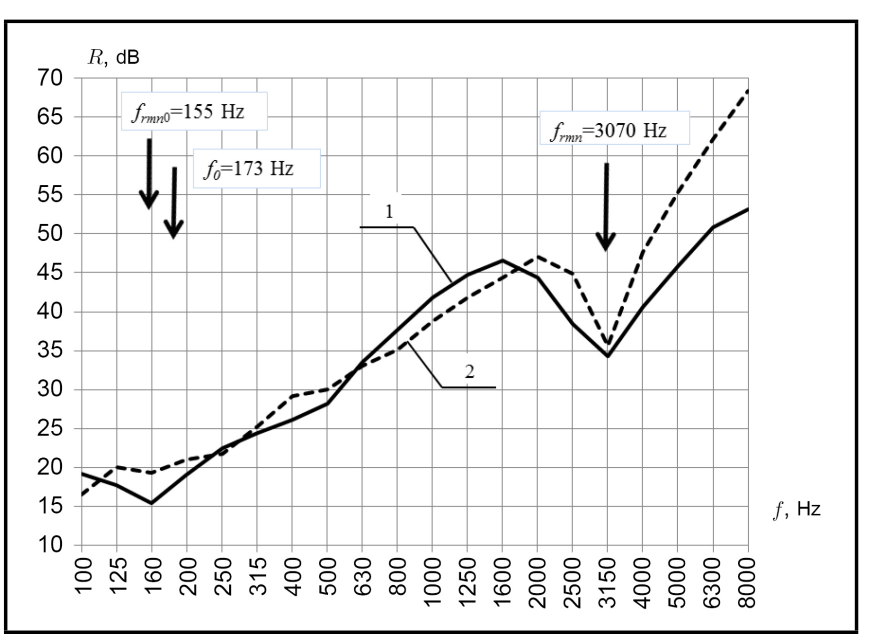

Figure 7. Frequency characteristics of sound insulation of double-glazed unit of $4+24+4 \mathrm{~mm}$ with edge damping of both glasses with use of material "BiMast Bomb" by a stripe of $20 \mathrm{~mm}$ along the perimeter: 1 - experimental data; 2 - theoretical calculation.

surement to measurement, so the effect of damping, which occurs during the installation of the structure, was always constant and was not reflected in the increase of sound insulation arising from the application of the edge damping.

The edge damping is only one factor affecting the sound insulation of translucent structures. When combined with other similar factors (including, for example, the number and thickness of glass, the width of the air gap, etc.) there can be expected further increase of sound insulation of the structure. The aim of the study on the current stage is to study the possibility of using the edge damping in particular to increase insulation, combine it with other factors to improve the result achieved - the goal of the next phase of the work.

In Fig. 6 there is a comparison of the theoretical calculation data in accordance with the above-mentioned method and experimental results for the construction without edge damping.

In Fig. 7 there is a comparison of the theoretical calculation data in accordance with the above-mentioned method and experimental results for the construction with edge damping of both glasses with use of material "BiMast Bomb" by a stripe of $20 \mathrm{~mm}$.

As can be seen from the comparison of frequency characteristics in Fig. 6 and Fig. 7, the theoretical calculation results quite accurately correspond to the experimental data within 


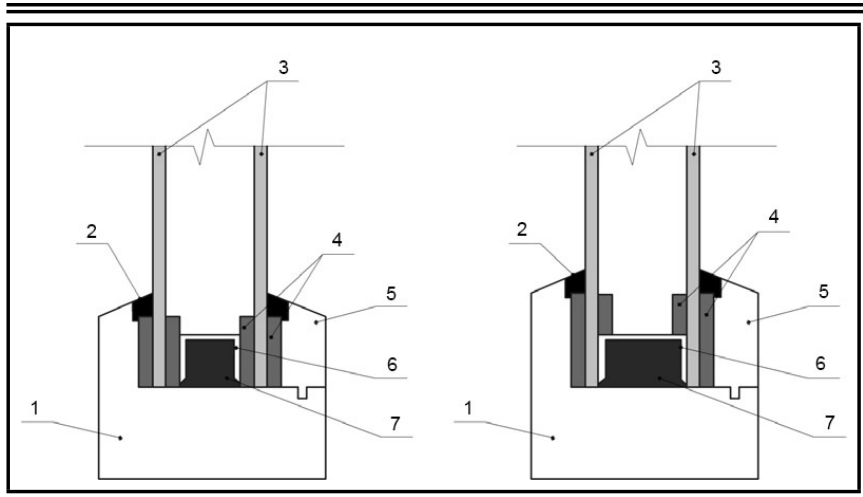

Figure 8. Examples of possible design solutions of windows using edge damping: 1 - the case of the window unit; 2 - rubber seal; 3 - silicate glass; 4 — vibration cushioning material; 5 - rubber sealing bead; 6 - spacer; 7 sealing mastic.

the whole standardized frequency range of $100-3150 \mathrm{~Hz}$ (i.e. within the areas of SSR (simple spatial resonance) and ISR (incomplete spatial resonances). Identification of the reasons for discrepancies between theoretical and experimental indices of sound insulation that are observed at frequencies higher than the standardized range (above $3150 \mathrm{~Hz}$, i.e. in the area of SSR) will be the goal of our further research.

\section{DESIGN SOLUTIONS OF WINDOWS WITH EDGE DAMPING}

Based on the above, as well as other experimental and theoretical data obtained by the staff of the NNGASU Laboratory of acoustics, design solutions of window units with IGUs with edge damping have been proposed in the course of studies of the capabilities of edge damping ${ }^{11,12}$ (see. Fig. 8). At that, it can be seen on the schemes that in this case it is possible to reduce the area of the translucent part of enclosure occupied by edge damping by placing the glazing parts coated with damping material inside the window unit housing.

An application for a patent for the invention was filed for the described method to improve sound insulation, as well as design solutions of structures based on it. ${ }^{13}$

\section{CONCLUSIONS}

Based on the results of the study, it can be concluded that the edge damping with opaque materials is a promising way to improve the sound insulation of windows and other glass structures. The increase of sound insulation due to the edge damping occurs in a wide range of frequencies of practically significant range (at frequencies of $315-8000 \mathrm{~Hz}$ for constructions of $4 \mathrm{~mm}$ glass). In the field of a resonance of structure as a system "mass-elasticity-mass" the extra edge damping is ineffective. This study established the most effective area of pasting the vibration damping material (1/8 of the glass area). In the future we plan to study the possibility of using regional damping in combination with other structural measures, allowing to increase the sound insulation of the structure.

\section{ACKNOWLEDGEMENTS}

The Reported study was Funded by Government Program of the Russian Federation "Development of science and technology" (2013-2020) within Program of Fundamental Researches of Ministry of Construction, Housing and Utilities of the Russian Federation and Russian Academy of Architecture and
Construction Sciences, section of scientific research projects No.7.6, project title: "Investigation of the resonant and inertial mechanism of sound transmission through new types of multilayer isotropic and orthotropic enclosing structures for unique buildings and development of theoretical methods for calculating their sound insulation".

\section{REFERENCES}

1 Murygin, D.V. Ways to improve sound insulation of translucent walling without significantly increasing its weight, textitPrivolzhskiy scientific journal, textbf1, 25-28, (2009).

2 Kochkin, A.A. Sound insulation of layered vibration dampened translucent walling, Building materials, textbf6, 4041, (2012).

3 Lilly, J.G. Recent Advances in Acoustical Glazing, textitSound and Vibration, 8-11, February 2004.

${ }^{4}$ Garg, N., Sharma, O., Maji, S. Experimental investigations on sound insulation through single, double \& triple window glazing for traffic noise abatement, textitJournal of Scientific \& Industrial Research, textbf70, 471-478, (2011).

5 Keller, U. Improved Sound Reduction with Laminated Glass. Glass processing days. Conference Proceedings, Tampere, Finland, 735-737, 18 to 21 June 2001.

6 Schimmelpenningh, J. Acoustic Interlayers for Laminated Glass âĂŞ What makes them different and how to estimate performance. Glass processing days. Conference Proceedings, 2-8, (2012).

7 Bogolepov, I.I. On the issue of sound insulation of transparent constructions. Sound attenuation: col. M: VTSNIIOT VTSSPS, 33-39, (1976).

8 Sedov, M.S. et al., textitSound Insulation. Engineering Acoustics of Vehicles: Handbook, N.I. Ivanov (ed.), Politekhnika, St. Petersburg, Ch. 1, 68-105, (1992).

9 ASTM E756-05(2010) «Standard Test Method for Measuring Vibration-Damping Properties of Materials»

10 ISO 10140-2 «Acoustics - Laboratory measurement of sound insulation of building elements - Part 2: Measurement of airborne sound insulation»

11 Puzankov, A.N., Schegolev, D.L. Investigation of the effect of the edge damping of translucent walling on its acoustic permeability, textitBuilding materials, textbf6, 36-37, (2012).

12 Puzankov, A.N. Study on effectiveness of usage of additional vibration cushioning to improve the sound insulation of modern windows, textitTGASU Herald, textbf2, 119129, (2015).

13 An application for a patent No. 2016102569 Russian Federation. A method for improving sound insulation of translucent structures / V.N. Bobylev, D.L. Schegolev, A.N. Puzankov; the applicant - Nizhny Novgorod State University of Architecture and Civil Engineering; appl. 01/26/2016. - 2016. - 1 p. 